\title{
Heavy flavour production at LHCb
}

\section{Fabio Ferrari ${ }^{* \dagger}$}

Università di Bologna and INFN

E-mail: fabio.ferrariecern.ch

The LHCb experiment is able to measure the production properties of heavy hadrons in a region that is complementary with respect to those of the ATLAS and CMS experiments. These measurements provide useful information to better understand quantum chromodynamics and to discriminate between theoretical models. The determination of the production cross-sections of $\Upsilon(1 S), \Upsilon(2 S), \Upsilon(3 S)$ in $p p$ collisions at a centre-of-mass energy of $13 \mathrm{TeV}$ is presented, together with the measurement of the production cross-section of $B^{+}$mesons in $p p$ collisions at centreof-mass energies of 7 and $13 \mathrm{TeV}$. Finally, the measurement of the $D_{s}^{+}$production asymmetry at $\sqrt{s}=7$ and $8 \mathrm{TeV}$ is also reported.

The International Conference on B-Physics at Frontier Machines - BEAUTY2018

6-11 May, 2018

La Biodola, Elba Island, Italy

* Speaker.

${ }^{\dagger}$ On behalf of the LHCb collaboration 


\section{Introduction}

Measurements of $\Upsilon$ production are a good probe of quantum chromodynamics (QCD) and can provide useful information about the hadronic production of heavy quarkonia. Several models have been proposed to discuss the underlying dynamics, such as the colour-singlet model (CSM) $[1,2,3$, $4,5,6,7]$ and non-relativistic QCD (NRQCD) [8, 9, 10]. The production properties of $\Upsilon(1 S), \Upsilon(2 S)$ and $\Upsilon(3 S)$ mesons have already been measured by LHCb at centre-of-mass energies of 2.76 [11], $7[12,13,14,15]$ and $8 \mathrm{TeV}[13,14,15]$.

The $B^{+}$production measurements provide an important test for the most recent calculations based on fixed next-to-leading order (NLO) QCD with next-to-leading logarithm (NLL) large transverse momentum resummation (FONLL) approach [16, 17]. The $B^{+}$production cross-section has been measured at different centre-of-mass energies by the CMS [18, 19], ATLAS [20] and LHCb [21, 22] collaborations.

At LHC energies about one in ten collisions produces a charmed hadron. The production rates of hadrons containing $c$ or $\bar{c}$ quarks (e.g. $D_{s}^{+}$and $D_{s}^{-}$mesons) are not expected to be equal in $p p$ collisions, as $c$ and $\bar{c}$ quarks may combine with $u$ and $d$ valence quarks from the beam remnant, and production asymmetries may arise as a consequence of this fact. A previous measurement of the $D_{s}^{+}$production asymmetry has been performed by the LHCb collaboration [23] using only data collected at a centre-of-mass energy of $7 \mathrm{TeV}$.

\section{2. $\Upsilon$ production in $p p$ collisions at $\sqrt{s}=13 \mathrm{TeV}$}

The data sample used corresponds to an integrated luminosity of $277 \mathrm{pb}^{-1}$ collected at a centreof-mass energy of $13 \mathrm{TeV}$ [24]. The $\Upsilon$ candidates are built from two muons with large momentum and transverse momentum. The two particles are also required to form a good quality vertex and to have an invariant mass in the range $8.5<M\left(\mu^{+} \mu^{-}\right)<11.5 \mathrm{GeV} / c^{2}$.

The double-differential production cross-section times dimuon branching fraction, $\mathscr{B}(\Upsilon \rightarrow$ $\left.\mu^{+} \mu^{-}\right)$, is defined as

$$
\frac{\mathrm{d}^{2} \sigma}{\mathrm{d} p_{\mathrm{T}} \mathrm{d} y} \times \mathscr{B}\left(\Upsilon \rightarrow \mu^{+} \mu^{-}\right)=\frac{N_{\mathrm{sig}}\left(p_{\mathrm{T}}, y\right)}{\mathscr{L} \times \varepsilon_{\mathrm{tot}}\left(p_{\mathrm{T}}, y\right) \times \Delta y \times \Delta p_{\mathrm{T}}},
$$

where $N_{\mathrm{sig}}\left(p_{\mathrm{T}}, y\right)$ is the signal yield in a given $\left(p_{\mathrm{T}}, y\right)$ bin, $\mathscr{L}$ is the integrated luminosity, $\varepsilon_{\mathrm{tot}}\left(p_{\mathrm{T}}, y\right)$ is the total efficiency as a function of $p_{\mathrm{T}}$ and $y$ and $\Delta p_{\mathrm{T}}$ and $\Delta y$ are the bin widths.

In order to obtain the signal yields, extended unbinned maximum likelihood fits to the dimuon invariant-mass distribution are performed in each bin. The signal components are described by three Crystal Ball functions, one for each $\Upsilon$, while the combinatorial background is modelled by an exponential function. The tail parameters of the Crystal Ball functions, the mass difference between the $\Upsilon$ states and the ratios of the resolution parameters are fixed to common values for all the fits. The dimuon invariant-mass distribution in the range $0<p_{\mathrm{T}}<30 \mathrm{GeV} / c$ and $2.0<y<4.5$ is shown in Fig. 1, with the results of the fit overlaid to the data points. The total number of signal events is $397841 \pm 796$ for $\Upsilon(1 S), 99790 \pm 469$ for $\Upsilon(2 S)$ and $50677 \pm 381$ for $\Upsilon(3 S)$.

The total efficiency is the product of several components. The detector acceptance, selection and trigger efficiencies are obtained from simulation. The tracking efficiency is obtained from 


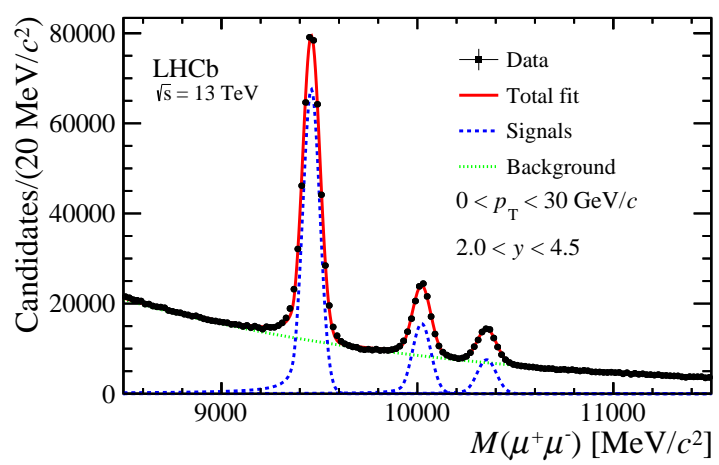

Figure 1: Dimuon invariant-mass distribution with the result of the fit overlaid to the data points.
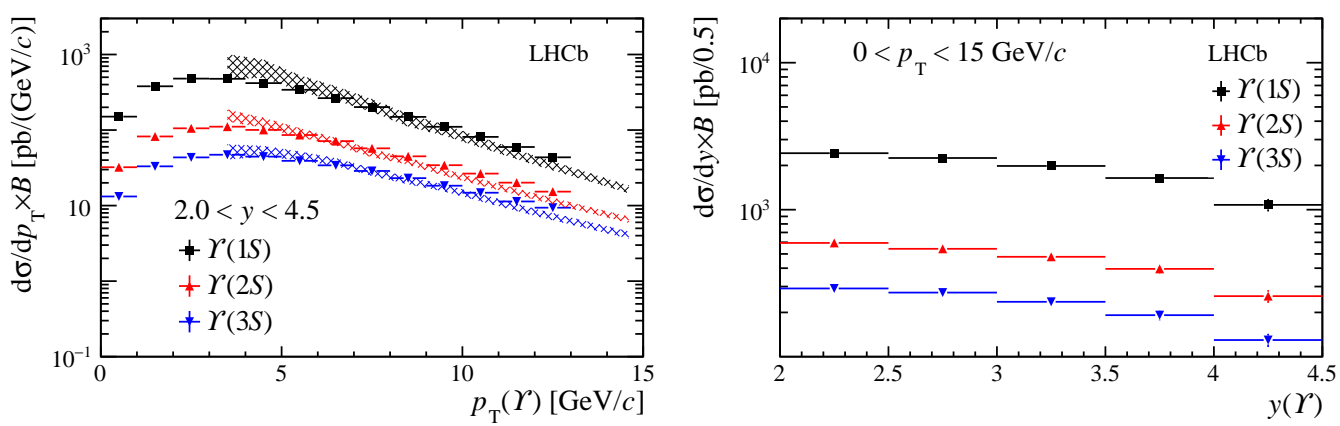

Figure 2: Single differential cross-sections times $\mathscr{B}\left(\Upsilon \rightarrow \mu^{+} \mu^{-}\right)$as a function of (left) $p_{\mathrm{T}}$ and (right) $y$ for (black squares) $\Upsilon(1 S)$, (red upward triangles) $\Upsilon(2 S)$ and (blue downward triangles) $\Upsilon(3 S)$. The shaded areas in the left plot represent the predictions from NRQCD.

simulation and corrected by means of a data-driven technique, while the particle identification (PID) efficiency is obtained from control samples of $J / \psi \rightarrow \mu^{+} \mu^{-}$and $\phi \rightarrow \mu^{+} \mu^{-}$decays using a tag-and-probe approach.

Several sources of systematic uncertainties related to the choice of the fit model, the determination of the efficiencies and the measurement of the integrated luminosity have been investigated.

The total cross-sections times $\mathscr{B}\left(\Upsilon \rightarrow \mu^{+} \mu^{-}\right)$in the range $0<p_{\mathrm{T}}<15 \mathrm{GeV} / c$ and $2.0<y<$ 4.5 are determined to be

$$
\begin{aligned}
& \mathscr{B}\left(\Upsilon(1 S) \rightarrow \mu^{+} \mu^{-}\right) \times \sigma(\Upsilon(1 S))=4687 \pm 10 \pm 294 \mathrm{pb}, \\
& \mathscr{B}\left(\Upsilon(2 S) \rightarrow \mu^{+} \mu^{-}\right) \times \sigma(\Upsilon(2 S))=1134 \pm 6 \pm 71 \mathrm{pb}, \\
& \mathscr{B}\left(\Upsilon(3 S) \rightarrow \mu^{+} \mu^{-}\right) \times \sigma(\Upsilon(3 S))=561 \pm 4 \pm 36 \mathrm{pb},
\end{aligned}
$$

where the first uncertainties are statistical and the second systematic. Integrating the doubledifferential cross-section over $p_{\mathrm{T}}(y)$, the results as a function of $y\left(p_{\mathrm{T}}\right)$ are obtained and are shown in Fig 2. The experimental data at high $p_{\mathrm{T}}$ are described well by the predictions from NRQCD [25].

\section{3. $B^{+}$production in $p p$ collisions at $\sqrt{s}=7$ and $13 \mathrm{TeV}$}

The data samples correspond to integrated luminosities of $1.0 \mathrm{fb}^{-1}$ and $0.3 \mathrm{fb}^{-1}$ at centre-of-mass 

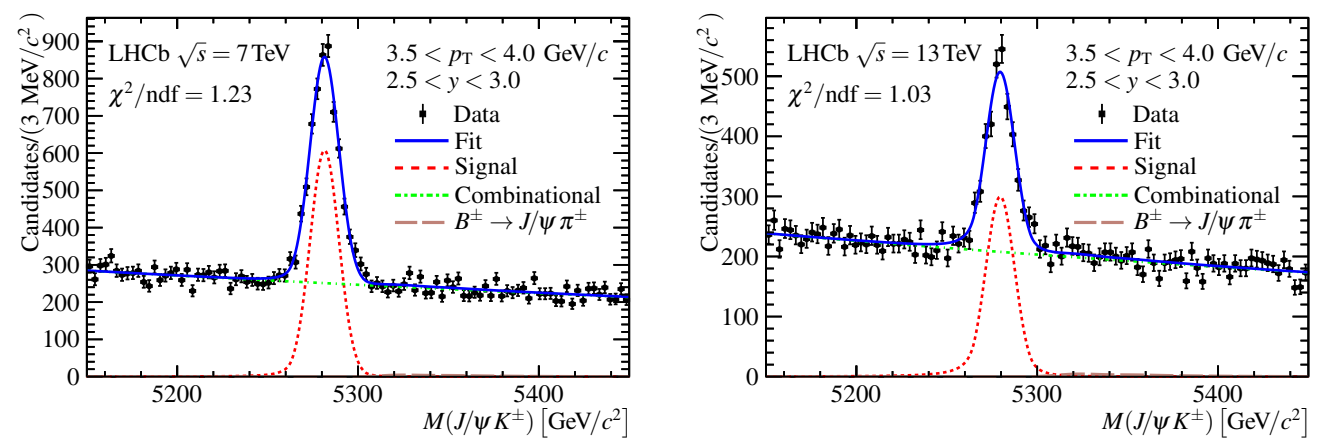

Figure 3: Invariant-mass distribution of $J / \psi K^{+}$candidates with $3.5<p_{\mathrm{T}}<4.0 \mathrm{GeV} / c$ and $2.5<y<3.0$ from data samples at the centre-of-mass energies of (left) 7 and (right) $13 \mathrm{TeV}$. The results of the fits to the $M\left(J / \psi K^{+}\right)$distributions are overlaid to the data points.

energies of 7 and $13 \mathrm{TeV}$, respectively. Dimuon candidates are built from muons with large transverse momentum, satisfying track quality and PID requirements. The muon pair is also required to form a common vertex and its invariant mass needs to be consistent with the known $J / \psi$ mass [26]. The $B^{+}$candidates are formed by combining $J / \psi$ and kaon candidates. The latter are required to have large transverse momenta $\left(p_{\mathrm{T}}\right)$ and to satisfy track quality criteria. No PID requirements are imposed on the kaons. The three tracks forming $B^{+}$candidates need to form a common vertex and to have a lifetime larger than $0.3 \mathrm{ps,} \mathrm{to} \mathrm{suppress} \mathrm{combinatorial} \mathrm{background} \mathrm{due} \mathrm{to} \mathrm{random}$ association of tracks from the primary vertex (PV).

The double-differential cross section is defined as

$$
\frac{\mathrm{d}^{2} \sigma}{\mathrm{d} p_{\mathrm{T}} \mathrm{d} y}=\frac{N_{\mathrm{sig}}\left(p_{\mathrm{T}}, y\right)}{\mathscr{L} \times \varepsilon_{\mathrm{tot}}\left(p_{\mathrm{T}}, y\right) \times \mathscr{B}\left(B^{+} \rightarrow J / \psi K^{+}\right) \times \mathscr{B}\left(J / \psi \rightarrow \mu^{+} \mu^{-}\right) \times \Delta y \times \Delta p_{\mathrm{T}}},
$$

where $N_{\text {sig }}\left(p_{\mathrm{T}}, y\right)$ is the number of signal candidates in a given $\left(p_{\mathrm{T}}, y\right)$ bin, $\mathscr{L}$ is the integrated luminosity, $\varepsilon\left(p_{\mathrm{T}}, y\right)$ is the total efficiency as a function of the $B^{+}$transverse momentum and rapidity, $\mathscr{B}\left(B^{+} \rightarrow J / \psi K^{+}\right)$is the branching ratio of $B^{+}$decays to $J / \psi K^{+}, \mathscr{B}\left(J / \psi \rightarrow \mu^{+} \mu^{-}\right)$is the branching ratio of $J / \psi$ decays to $\mu^{+} \mu^{-}$[26] and $\Delta y$ and $\Delta p_{\mathrm{T}}$ are the bin widths. The value $\mathscr{B}\left(B^{+} \rightarrow J / \psi K^{+}\right)=(1.044 \pm 0.040) \times 10^{-3}$ is obtained by combining previous determinations from Belle [28] and BaBar [29] collaborations.

The signal yield in each kinematic bin is obtained by means of extended unbinned maximum likelihood fits to the $J / \psi K^{+}$invariant mass $\left(M\left(J / \psi K^{+}\right)\right)$. The probability density function consists in a modified Crystal Ball (CB) function [27] describing the signal component, an exponential function modelling the combinatorial background and double CB function parameterising the contamination from the Cabibbo suppressed $B^{+} \rightarrow J / \psi \pi^{+}$decay mode. The $M\left(J / \psi K^{+}\right)$distribution in one $\left(p_{\mathrm{T}}, y\right)$ bin at different centre-of-mass energies is shown in Fig. 3, with the result of the fits overlaid to the data points.

The total efficiency is the product of several components. The detector acceptance, particle reconstruction and selection efficiencies are determined from simulation, whereas the muons PID and tracking efficiencies are obtained from a control data sample of $J / \psi \rightarrow \mu^{+} \mu^{-}$decays. The trigger efficiency is estimated with both data-driven techniques and simulation. 

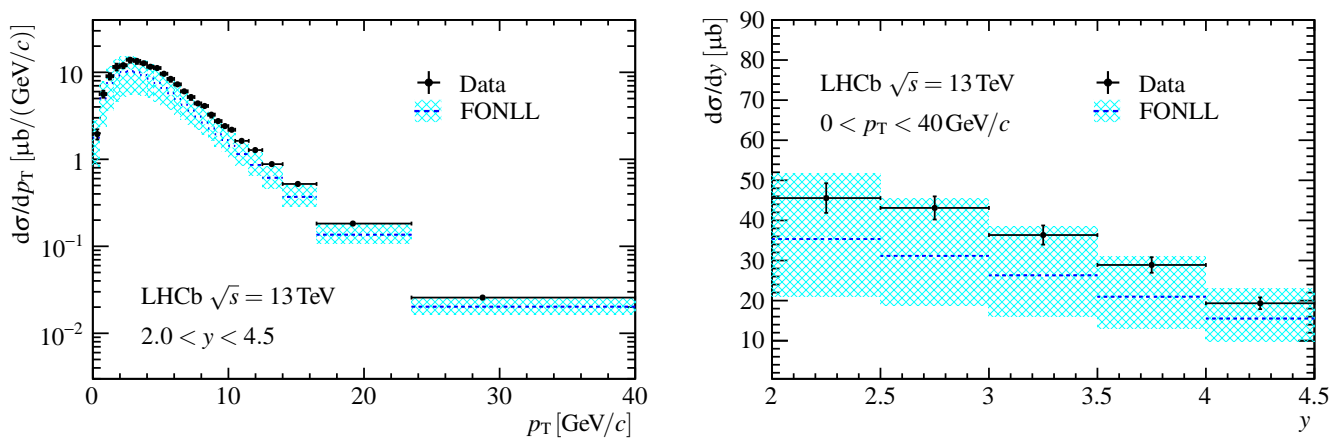

Figure 4: Differential production cross-section of $B^{+}$candidates as a function of (left) $p_{\mathrm{T}}$ and (right) $y$ for $13 \mathrm{TeV}$ data. The shaded areas represent the FONLL predictions.
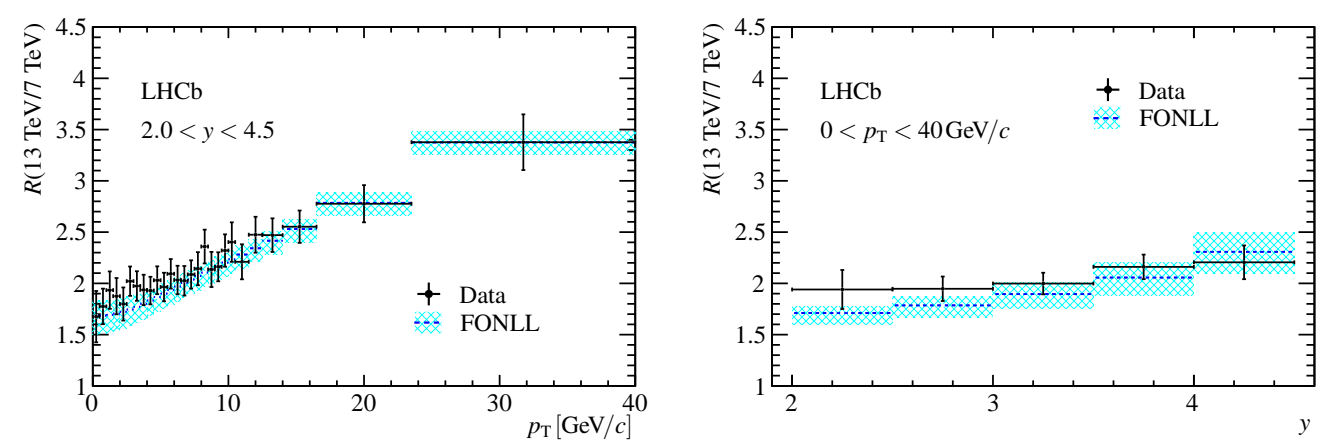

Figure 5: Cross-section ratio as a function of (left) $p_{\mathrm{T}}$ and (right) $y$. The shaded areas represent the FONLL predictions.

Several sources of systematic uncertainties related to the luminosity determination, branching fractions, signal yields and efficiencies have been investigated.

The production cross-sections of $B^{+}$mesons integrated in the range $0<p_{\mathrm{T}}<40 \mathrm{GeV} / c$ and $2.0<y<4.5$ for 7 and $13 \mathrm{TeV}$ data are found to be

$$
\begin{gathered}
\sigma\left(p p \rightarrow B^{+} X\right)_{\sqrt{s}=7 \mathrm{TeV}}=43.0 \pm 0.2 \pm 2.5 \pm 1.7 \mu \mathrm{b}, \\
\sigma\left(p p \rightarrow B^{+} X\right)_{\sqrt{s}=13 \mathrm{TeV}}=86.6 \pm 0.5 \pm 5.4 \pm 3.4 \mu \mathrm{b},
\end{gathered}
$$

where the first uncertainties are statistical, the second are systematic and the third are due to the limited knowledge of $\mathscr{B}\left(B^{+} \rightarrow J / \psi K^{+}\right)$.

Integrating the double-differential cross-sections over $p_{\mathrm{T}}(y)$, the cross-section as a function of $y\left(p_{\mathrm{T}}\right)$ is obtained. The distributions obtained from $13 \mathrm{TeV}$ data are shown in Fig. 4. Taking the ratio between the results obtained at the two different centre-of-mass energies, most of the systematic uncertainties cancel out. The obtained ratios as a function of $p_{\mathrm{T}}$ and $y$ are shown in Fig. 5. All results show good agreement with FONLL predictions [30] over the measured range.

\section{4. $D_{s}^{+}$production asymmetry at $\sqrt{s}=7$ and $8 \mathrm{TeV}$}

The data sample corresponds to integrated luminosities of $1.0 \mathrm{fb}^{-1}$ and $2.0 \mathrm{fb}^{-1}$ at centre-of-mass 

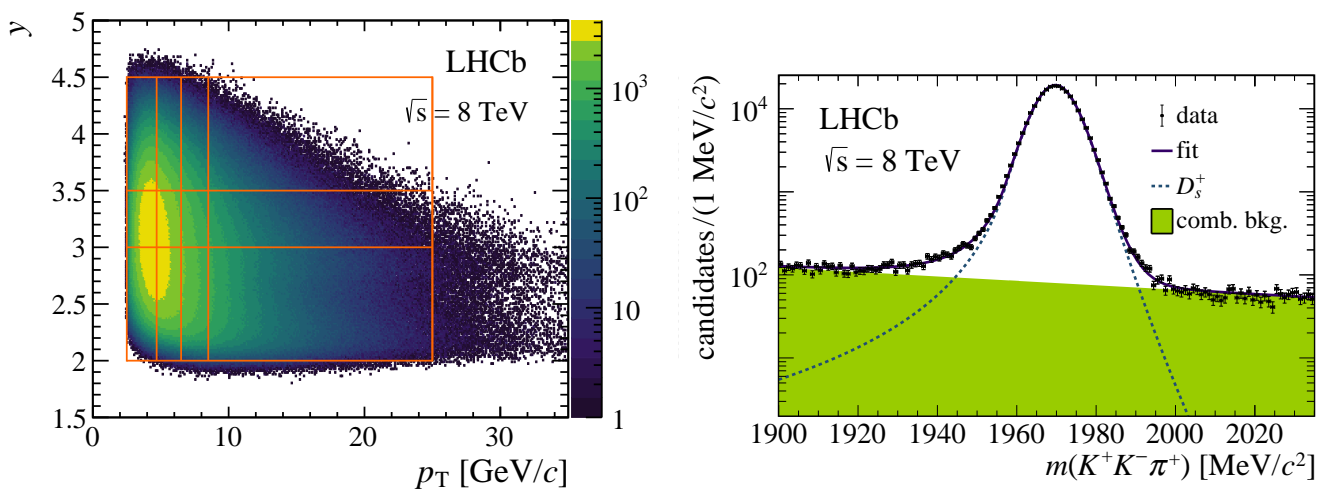

Figure 6: (left) Distribution of rapidity versus transverse momentum for $D_{s}^{ \pm}$candidates. The binning scheme used to measure the production asymmetry in different phase-space regions is also shown. (right) Invariantmass distribution of $K^{+} K^{-} \pi^{+}$candidates in the range $2.5<p_{\mathrm{T}}<4.7 \mathrm{GeV} / c$ and $2.0<y<3.0$. The results of the fit are overlaid to the data points.

energies of 7 and $8 \mathrm{TeV}$, respectively. The $D_{s}^{+}$candidates are reconstructed in the $\phi\left(\rightarrow K^{+} K^{-}\right) \pi^{+}$ final state. The three tracks forming the $D_{s}^{+}$meson are required not to point back to any PV and to have high momenta and transverse momenta. The $D_{s}^{+}$candidates need to point to the PV and their decay vertices have to be significantly displaced from any PV. Kaons and pions are selected using PID criteria and the invariant mass of the kaon pair is required to be within $20 \mathrm{MeV} / \mathrm{c}^{2}$ of the known $\phi$ mass [26].

Assuming that $C P$ violation in the Cabibbo favoured $D_{s}^{+} \rightarrow \phi\left(\rightarrow K^{+} K^{-}\right) \pi^{+}$decay is negligible at the level of precision of this measurement, the production asymmetry can be obtained as

$$
A_{\mathrm{P}}=\frac{1}{1-f_{\mathrm{bkg}}}\left(A_{\mathrm{raw}}-A_{\mathrm{D}}-f_{\mathrm{bkg}} A_{\mathrm{sec}}\right)
$$

where $f_{\mathrm{bkg}}$ is the fraction of $D_{s}^{+}$mesons produced from a $b$-hadron decay, $A_{\text {raw }}$ is the raw asymmetry, $A_{\mathrm{D}}$ is the sum of various detection and instrumental asymmetries and $A_{\mathrm{sec}}$ is the production asymmetry of $b$-hadrons decaying to $D_{s}^{+}$mesons. The raw asymmetry is defined as

$$
A_{\text {raw }}=\frac{N\left(D_{s}^{+}\right)-N\left(D_{s}^{-}\right)}{N\left(D_{s}^{+}\right)+N\left(D_{s}^{-}\right)}
$$

where $N\left(D_{s}^{+}\right)\left(N\left(D_{s}^{-}\right)\right)$is the observed number of $D_{s}^{+}\left(D_{s}^{-}\right)$signal candidates. The production asymmetry is measured in two-dimensional bins of transverse momentum and rapidity to check if it exhibits any dependence on these quantities. The $\left(p_{\mathrm{T}}, \eta\right)$ distribution of $D_{s}^{+}$candidates for $8 \mathrm{TeV}$ data is shown in the left part of Fig. 6, with the chosen binning scheme overlaid.

The raw asymmetry is measured by means of binned maximum-likelihood fits to the invariant mass of the $K^{+} K^{-} \pi^{+}$final state. The signal component is modelled using an Hypathia function [31], while the combinatorial background is described by an exponential function. The distribution of the $K^{+} K^{-} \pi^{+}$invariant mass in the range $2.5<p_{\mathrm{T}}<4.7 \mathrm{GeV} / c$ and $2.0<y<3.0$ is shown in the right part of Fig. 6, with the result of the fit overlaid to the data points.

The detection asymmetry is the sum of various components as tracking, PID and trigger asymmetries. All of these are obtained by means of data-driven techniques. The fraction of secondary 

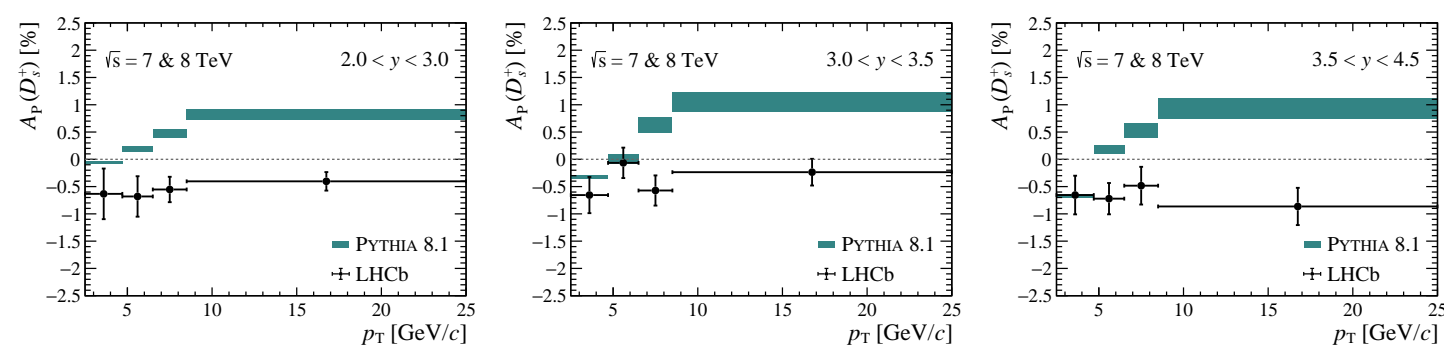

Figure 7: Production asymmetry of $D_{s}^{+}$candidates as a function of the transverse momentum in the range (left) $2.0<y<3.0$, (middle) $3.0<y<3.5$ and (right) $3.5<y<4.5$. The shaded areas represent the production asymmetry obtained from the PYTHIA 8.1 event generator.

$D_{s}^{+}$decays is obtained from simulation, cross-sections [22, 32] and branching fractions [26] measurements and it is found to be $f_{\mathrm{bkg}}=(4.12 \pm 1.23) \%$. The production asymmetries of $b$-hadrons are taken from external inputs $[33,34,35]$.

Several sources of systematic uncertainties related to the choice of the fit model and to the determination of the detection asymmetries have been investigated.

The $D_{s}^{+}$production asymmetry integrated in the range $2.5<p_{\mathrm{T}}<25 \mathrm{GeV} / c$ and $2.0<y<4.5$ is found to be

$$
A_{\mathrm{P}}=(-0.52 \pm 0.13 \pm 0.10) \%,
$$

where the first uncertainty is statistical and the second systematic. A deviation of $3.3 \sigma$ from the hypothesis of no production asymmetry is found.

The production asymmetry is also measured as a function of the transverse momentum and rapidity of the $D_{s}^{+}$candidates. The results are shown in Fig. 7, together with the prediction obtained from the PYTHIA 8.1 event generator $[36,37]$. The simulated events show a strong dependence on the $D_{s}^{+}$transverse momentum that is not observed in data.

\section{Conclusions}

Thanks to its unique forward geometry, the LHCb experiment is able to measure heavy-hadron production in a kinematic region that is complementary to those of ATLAS and CMS. The determination of the production cross-section of $\Upsilon(1 S), \Upsilon(2 S)$ and $\Upsilon(3 S)$ mesons at $\sqrt{s}=13 \mathrm{TeV}$ is reported and found to be in agreement with the prediction from NRQCD in the high $p_{\mathrm{T}}$ region. The measurement of the production cross-section of $B^{+}$mesons collected at centre-of-mass energies of 7 and $13 \mathrm{TeV}$ is also presented and the results obtained are in agreement with FONLL predictions. Finally, a measurement of the $D_{s}^{+}$production asymmetry with 7 and $8 \mathrm{TeV}$ data is reported and a deviation of $3.3 \sigma$ from the hypothesis of no production asymmetry is found. No dependence of the production asymmetry on the candidates transverse momentum is observed, in contrast with the predictions of the PYTHIA 8.1 event generator.

The LHCb experiment is playing a key role in the quest for a better knowledge of heavy flavour production mechanisms. The results here presented can be utilised to discriminate between several theoretical models and can provide useful inputs to tune the production mechanisms in event generators. 


\section{References}

[1] C. E. Carlson and R. Suaya, Hadronic production of $\psi / J$ mesons, Phys. Rev. D 14 (1976) 3115.

[2] A. Donnachie and P. V. Landshoff, Production of lepton pairs, J/ $\psi$ and charm with hadron beams, Nucl. Phys. B 112 (1976) 233.

[3] S. D. Ellis, M. B. Einhorn and C. Quigg, Comment on hadronic production of psions, Phys. Rev. Lett. 36 (1976) 1263.

[4] H. Fritzsch, Producing heavy quark flavors in hadronic collisions: a test of quantum chromodynamics, Phys. Lett. 67B (1977) 217.

[5] M. Gluck, J. F. Owens and E. Reya, Gluon contribution to hadronic J/ $\psi$ production, Phys. Rev. D 17 (1978) 2324.

[6] C. H. Chang, Hadronic production of J/ $\psi$ associated with a gluon, Nucl. Phys. B 172 (1980) 425.

[7] R. Baier and R. Ruckl, Hadronic production of $J / \psi$ and $\Upsilon$ : transverse momentum distributions, Phys. Lett. 102B (1981) 364.

[8] G. T. Bodwin, E. Braaten and G. P. Lepage, Rigorous QCD analysis of inclusive annihilation and production of heavy quarkonium, Phys. Rev. D 51 (1995) 1125 Erratum: [Phys. Rev. D 55 (1997) 5853] [hep-ph/9407339].

[9] P. L. Cho and A. K. Leibovich, Color octet quarkonia production, Phys. Rev. D 53 (1996) 150 [hep-ph/9505329].

[10] P. L. Cho and A. K. Leibovich, Color octet quarkonia production 2, Phys. Rev. D 53 (1996) 6203 [hep-ph/9511315].

[11] R. Aaij et al. [LHCb Collaboration], Measurement of $\Upsilon$ production in pp collisions at $\sqrt{s}=2.76 \mathrm{TeV}$, Eur. Phys. J. C 74 (2014) no.4, 2835 [arXiv:1402.2539 [hep-ex]].

[12] R. Aaij et al. [LHCb Collaboration], Measurement of $\Upsilon$ production in pp collisions at $\sqrt{s}=7 \mathrm{TeV}$, Eur. Phys. J. C 72 (2012) 2025 [arXiv:1202.6579 [hep-ex]].

[13] R. Aaij et al. [LHCb Collaboration], Measurement of the exclusive $\Upsilon$ production cross-section in pp collisions at $\sqrt{s}=7 \mathrm{TeV}$ and $8 \mathrm{TeV}$, JHEP 1509 (2015) 084 [arXiv:1505.08139 [hep-ex]].

[14] R. Aaij et al. [LHCb Collaboration], Forward production of $\Upsilon$ mesons in pp collisions at $\sqrt{s}=7$ and $8 \mathrm{TeV}$, JHEP 1511 (2015) 103 [arXiv:1509.02372 [hep-ex]].

[15] R. Aaij et al. [LHCb Collaboration], Measurement of the $\Upsilon$ polarizations in pp collisions at $\sqrt{s}=7$ and 8 TeV," JHEP 1712 (2017) 110 [arXiv:1709.01301 [hep-ex]].

[16] M. Cacciari, M. Greco and P. Nason, The $p_{\mathrm{T}}$ spectrum in heavy flavor hadroproduction, JHEP 9805 (1998) 007 [hep-ph/9803400].

[17] M. Cacciari, S. Frixione and P. Nason, The $p_{\mathrm{T}}$ spectrum in heavy flavor photoproduction, JHEP 0103 (2001) 006 [hep-ph/0102134].

[18] V. Khachatryan et al. [CMS Collaboration], Measurement of the $B^{+}$Production Cross Section in pp Collisions at $\sqrt{s}=7 \mathrm{TeV}$, Phys. Rev. Lett. 106 (2011) 112001 [arXiv:1101.0131 [hep-ex]].

[19] V. Khachatryan et al. [CMS Collaboration], Measurement of the total and differential inclusive $B^{+}$ hadron cross sections in pp collisions at $\sqrt{s}=13 \mathrm{TeV}$, Phys. Lett. B 771 (2017) 435 [arXiv:1609.00873 [hep-ex]]. 
[20] G. Aad et al. [ATLAS Collaboration], Measurement of the differential cross-section of $B^{+}$meson production in pp collisions at $\sqrt{s}=7 \mathrm{TeV}$ at ATLAS, JHEP 1310 (2013) 042 [arXiv:1307.0126 [hep-ex]].

[21] R. Aaij et al. [LHCb Collaboration], Measurement of the $B^{ \pm}$production cross-section in pp collisions at $\sqrt{s}=7 \mathrm{TeV}$, JHEP 1204 (2012) 093 [arXiv:1202.4812 [hep-ex]].

[22] R. Aaij et al. [LHCb Collaboration], Measurement of B meson production cross-sections in proton-proton collisions at $\sqrt{s}=7 \mathrm{TeV}$, JHEP 1308 (2013) 117 [arXiv:1306.3663 [hep-ex]].

[23] R. Aaij et al. [LHCb Collaboration], Measurement of the $D_{s}^{+}-D_{s}^{-}$production asymmetry in $7 \mathrm{TeV} p p$ collisions, Phys. Lett. B 713 (2012) 186 [arXiv:1205.0897 [hep-ex]].

[24] R. Aaij et al. [LHCb Collaboration], Measurement of $\Upsilon$ production in pp collisions at $\sqrt{s}=13 \mathrm{TeV}$, JHEP 1807 (2018) 134 [arXiv:1804.09214 [hep-ex]].

[25] Y. Feng, B. Gong, L. P. Wan and J. X. Wang, An updated study of $\Upsilon$ production and polarization at the Tevatron and LHC, Chin. Phys. C 39 (2015) no.12, 123102 [arXiv:1503.08439 [hep-ph]].

[26] M. Tanabashi et al. [Particle Data Group], Review of particle physics, Phys. Rev. D 98 (2018) 030001.

[27] T. Skwarnicki, A study of the radiative cascade transitions between the $\Upsilon^{\prime}$ and $\Upsilon$ resonances, DESY-F31-86-02.

[28] K. Abe et al. [Belle Collaboration], Measurement of branching fractions and charge asymmetries for two-body B meson decays with charmonium, Phys. Rev. D 67 (2003) 032003 [hep-ex/0211047].

[29] B. Aubert et al. [BaBar Collaboration], Measurement of branching fractions and charge asymmetries for exclusive B decays to charmonium, Phys. Rev. Lett. 94 (2005) 141801 [hep-ex/0412062].

[30] M. Cacciari, M. L. Mangano and P. Nason, Gluon PDF constraints from the ratio of forward heavy-quark production at the LHC at $\sqrt{s}=7$ and $13 \mathrm{TeV}$, Eur. Phys. J. C 75 (2015) no.12, 610 [arXiv:1507.06197 [hep-ph]].

[31] D. Martínez Santos and F. Dupertuis, Mass distributions marginalized over per-event errors, Nucl. Instrum. Meth. A 764 (2014) 150 [arXiv:1312.5000 [hep-ex]].

[32] R. Aaij et al. [LHCb Collaboration], Study of the kinematic dependences of $\Lambda_{b}^{0}$ production in pp collisions and a measurement of the $\Lambda_{b}^{0} \rightarrow \Lambda_{c}^{+} \pi^{-}$branching fraction, JHEP 1408 (2014) 143 [arXiv:1405.6842 [hep-ex]].

[33] R. Aaij et al. [LHCb Collaboration], Measurement of the $B^{ \pm}$production asymmetry and the $C P$ asymmetry in $B^{ \pm} \rightarrow J / \psi K^{ \pm}$decays, Phys. Rev. D 95 (2017) no.5, 052005 [arXiv:1701.05501 [hep-ex]].

[34] R. Aaij et al. [LHCb Collaboration], Measurement of $B^{0}, B_{s}^{0}, B^{+}$and $\Lambda_{b}^{0}$ production asymmetries in 7 and 8 TeV proton-proton collisions, Phys. Lett. B 774 (2017) 139 [arXiv:1703.08464 [hep-ex]].

[35] R. Aaij et al. [LHCb Collaboration], Measurement of the semileptonic CP asymmetry in $B^{0}-\bar{B}^{0}$ mixing, Phys. Rev. Lett. 114 (2015) 041601 [arXiv:1409.8586 [hep-ex]].

[36] T. Sjostrand, S. Mrenna and P. Z. Skands, PYTHIA 6.4 Physics and Manual, JHEP 0605 (2006) 026 [hep-ph/0603175].

[37] T. Sjostrand, S. Mrenna and P. Z. Skands, A Brief Introduction to PYTHIA 8.1, Comput. Phys. Commun. 178 (2008) 852 [arXiv:0710.3820 [hep-ph]]. 